\title{
A Delay Decomposition Approach to the Stability Analysis of Singular Systems with Interval Time-Varying Delay
}

\author{
Jianmin Jiao and Rui Zhang \\ Department of Mathematics, Baoji University of Arts and Sciences, Baoji 721013, China \\ Correspondence should be addressed to Jianmin Jiao; jmjiao@126.com
}

Received 4 May 2014; Revised 25 June 2014; Accepted 26 June 2014; Published 13 July 2014

Academic Editor: Yuxin Zhao

Copyright (C) 2014 J. Jiao and R. Zhang. This is an open access article distributed under the Creative Commons Attribution License, which permits unrestricted use, distribution, and reproduction in any medium, provided the original work is properly cited.

\begin{abstract}
This paper investigates delay-dependent stability problem for singular systems with interval time-varying delay. An appropriate Lyapunov-Krasovskii functional is constructed by decomposing the delay interval into multiple equidistant subintervals, where both the information of every subinterval and time-varying delay have been taken into account. Employing the LyapunovKrasovskii functional, improved delay-dependent stability criteria for the considered systems to be regular, impulse-free, and stable are established. Finally, two numerical examples are presented to show the effectiveness and less conservativeness of the proposed method.
\end{abstract}

\section{Introduction}

During the last few decades, there has been a growing research interest in the analysis and synthesis of time-delay systems, which widely exist in various practical systems such as biological systems, chemical systems, electronic systems, and network control systems [1]. Usually, the range of delays considered in most of existing references is from zero to an upper bound [2-6]. In practice, however, the delay range may have a nonzero lower bound, and such systems are referred to as interval time-varying delay systems. For this reason, the stability of the systems with such interval time-varying delays has attracted considerable attention. As we know, in order to reduce conservatism of the stability criteria, many approaches were developed; for example, by using free-weighting matrices, $[7,8]$ present some stability conditions for systems with interval time-varying delays. In [9], via a Lyapunov-Krasovskii functional with fewer matrix variables whose derivative is estimated using the convex analysis method, a simple stability criterion was obtained; this result is improved in [10] by using the reciprocally convex approach. Recently, some new methods were proposed to the stability analysis of interval time-varying delay systems. New classes of Lyapunov-Krasovskii functionals and augmented Lyapunov-Krasovskii functionals were introduced in [1115], where some multi-integral terms were introduced in
Lyapunov-Krasovskii functionals. In [16-18], combining with a decomposition approach, the upper bound of the derivative of Lyapunov-Krasovskii functional was estimated tightly and new stability results were obtained. It has been shown that these new methods can be applied efficiently to derive less conservative stability results for systems with interval timevarying delay.

On the other hand, singular systems have been extensively studied in the past years due to the fact that singular systems can better describe the behavior of some physical systems than regular ones. Singular systems are also referred to as descriptor systems, differential algebraic systems, or semistate systems. A great number of fundamental results based on the theory of regular systems have been extended to the area of singular systems [19]. It is well known that the stability analysis for singular systems is much more complicated than that for regular systems because it requires considering not only stability but also regularity and absence of impulse (for continuous singular systems) [20-30] or causality (for discrete singular systems) [31-34]. Recently, more and more attention has been paid to singular systems with delay. To obtain delay-dependent conditions, many efforts have been made in the literature. Via different Lyapunov-Krasovskii functionals, some stability criteria were obtained in [20, $21]$, and the results in $[20,21]$ were improved in $[22,23]$ 
using the free-weighting matrices and discretized LyapunovKrasovskii functional, respectively. However, the involved time delays in [20-23] are all time invariant, which limits the scope of applications of the given results. In the case where time-varying delays appear in singular systems, some stability results were proposed in [24-27]. The range of time-varying delay considered in [24-27] is from zero to an upper bound. For singular systems with interval time-varying delay, there are fewer results [28-30] and there still exists some room for further investigation.

In this paper, our purpose is to present some new delaydependent stability criteria for singular systems with interval time-varying delay. An appropriate Lyapunov-Krasovskii functional is constructed by using the idea of "delay decomposition." Employing this Lyapunov-Krasovskii functional, some new delay-dependent sufficient conditions ensuring the stability for the considered systems are obtained in terms of LIMs. The main contribution of this paper is in two aspects. First, the new results will be less conservative than the existing ones, which will be demonstrated by two numerical examples. Second, the new results will involve fewer decision variables than some existing ones; hence, they are mathematically less complex and more computationally efficient.

Notations. Throughout this paper, $\mathbf{R}^{n}$ denotes the $n$ dimensional Euclidean space, while $\mathbf{R}^{m \times n}$ refers to the set of all real matrices with $m$ rows and $n$ columns. $A^{T}$ represents the transpose of the matrix $A$. For real symmetric matrices $X$ and $Y$, the notation $X \geqslant Y$ (resp., $X>Y$ ) means matrix $X-Y$ is positive-semidefinite (resp., positive-definite). $I$ is the identity matrix with appropriate dimensions. $\|x\|$ refers to the Euclidean norm of the vector $x$; that is, $\|x\|=\sqrt{x^{T} x}$.

\section{Problem Formulation and Preliminaries}

Consider the following singular system with interval timevarying delay described by

$$
\begin{aligned}
E \dot{x}(t) & =A x(t)+A_{d} x(t-d(t)), \\
x(t) & =\varphi(t), \quad t \in\left[-d_{2}, 0\right],
\end{aligned}
$$

where $x(t) \in \mathbf{R}^{n}$ is the state vector and the initial condition $\varphi(t) \in \mathbf{R}^{n}$ is a continuously differentiable vector-valued function. The matrix $E \in \mathbf{R}^{n \times n}$ may be singular and ranks $E=r \leqslant n, A, A_{d} \in \mathbf{R}^{n \times n}$ are known real constant matrices. $d(t)$ is the time-varying delay satisfying

$$
d_{1} \leqslant d(t) \leqslant d_{2}, \quad \dot{d}(t) \leqslant \mu,
$$

where $0<d_{1}<d_{2}$ and $0 \leqslant \mu<1$ are known constant scalars.

In this paper, our objective is to establish new delaydependent stability conditions for singular time-delay system (1). The following definitions and lemma will be used in the proof of our main results.

Definition 1 (see [19]). (i) The pair $(E, A)$ is said to be regular if $\operatorname{det}(s E-A)$ is not identically zero. (ii) The pair $(E, A)$ is said to be impulse-free if $\operatorname{deg}(\operatorname{det}(s E-A))=\operatorname{rank} E$.
Definition 2 (see $[28,30])$. (i) The singular time-delay system (1) is said to be regular and impulse-free if the pair $(E, A)$ is regular and impulse-free. (ii) The singular time-delay system (1) is said to be stable if, for any $\varepsilon>0$, there exists a scalar $\delta(\varepsilon)>0$ such that, for any compatible initial conditions $\varphi(t)$ satisfying $\sup _{-d_{2} \leqslant t \leqslant 0}\|\varphi(t)\| \leqslant \delta(\varepsilon)$, the solution $x(t)$ of system (1) satisfies $\|x(t)\| \leqslant \varepsilon$ for any $t \geqslant 0$; moreover, $\lim _{t \rightarrow \infty} x(t)=$ 0 .

Lemma 3 (see [1]). For any symmetric positive defined matrix $R>0$, scalars $\gamma_{2}>\gamma_{1} \geqslant 0$, and vector function $x:\left[\gamma_{1}, \gamma_{2}\right] \mapsto$ $\mathbf{R}^{n}$ such that the following integrations are well defined, the following inequality holds

$$
-\left(\gamma_{2}-\gamma_{1}\right) \int_{\gamma_{1}}^{\gamma_{2}} x^{T}(s) R x(s) \mathrm{d} s \leqslant-\int_{\gamma_{1}}^{\gamma_{2}} x^{T}(s) \mathrm{d} s R \int_{\gamma_{1}}^{\gamma_{2}} x(s) \mathrm{d} s .
$$

\section{Main Results}

In this section, we consider the stability problem of singular time-delay system (1). We first present a delay-dependent stability criterion for singular time-delay system (1) as follows.

Theorem 4. Given scalars $0<d_{1}<d_{2}$ and $0 \leqslant \mu<1$ for any delay $d(t)$ satisfying (2), singular time-delay system (1) is regular, impulse-free, and stable if there exist the following matrices:

$$
\begin{gathered}
P>0, \quad W>0, \quad Q=\left[\begin{array}{ll}
Q_{1} & Q_{2} \\
Q_{2}^{T} & Q_{3}
\end{array}\right]>0, \\
R=\left[\begin{array}{ll}
R_{1} & R_{2} \\
R_{2}^{T} & R_{3}
\end{array}\right]>0, \quad S_{i}>0, \\
Z_{i}>0, \quad M_{i}(i=1,2),
\end{gathered}
$$

and $U$, such that the LMIs

$$
\begin{gathered}
\Xi+\Xi_{k}<0, \\
{\left[\begin{array}{cc}
Z_{k} & M_{k} \\
M_{k}^{T} & Z_{k}
\end{array}\right]>0}
\end{gathered}
$$

hold for any $k \in\{1,2\}$, where

$$
\begin{aligned}
\Xi= & e_{1}\left(E^{T} P+U \Psi^{T}\right) e_{0}^{T}+e_{0}\left(P E+\Psi U^{T}\right) e_{1}^{T} \\
& +e_{0}\left(\left(\frac{d_{1}}{2}\right)^{2}\left(S_{1}+S_{2}\right)+\left(\frac{d_{2}-d_{1}}{2}\right)^{2}\left(Z_{1}+Z_{2}\right)\right) e_{0}^{T} \\
& +e_{3} W e_{3}^{T}-(1-\mu) e_{6} W e_{6}^{T}+\left[\begin{array}{ll}
e_{1} & e_{2}
\end{array}\right] Q\left[\begin{array}{ll}
e_{1} & e_{2}
\end{array}\right]^{T}
\end{aligned}
$$




$$
\begin{aligned}
& -\left[\begin{array}{ll}
e_{2} & e_{3}
\end{array}\right] Q\left[\begin{array}{ll}
e_{2} & e_{3}
\end{array}\right]^{T}+\left[\begin{array}{ll}
e_{3} & e_{4}
\end{array}\right] R\left[\begin{array}{ll}
e_{3} & e_{4}
\end{array}\right]^{T} \\
& -\left[\begin{array}{ll}
e_{4} & e_{5}
\end{array}\right] R\left[\begin{array}{ll}
e_{4} & e_{5}
\end{array}\right]^{T}-\left(e_{1}-e_{2}\right) E^{T} S_{1} E\left(e_{1}-e_{2}\right)^{T} \\
& -\left(e_{2}-e_{3}\right) E^{T} S_{2} E\left(e_{2}-e_{3}\right)^{T}, \\
\Xi_{1}= & -\left(e_{4}-e_{5}\right) E^{T} Z_{2} E\left(e_{4}-e_{5}\right)^{T} \\
& -\left[\begin{array}{c}
e_{6}^{T}-e_{4}^{T} \\
e_{3}^{T}-e_{6}^{T}
\end{array}\right]^{T}\left[\begin{array}{ll}
E^{T} Z_{1} E & E^{T} M_{1} E \\
E^{T} M_{1}^{T} E & E^{T} Z_{1} E
\end{array}\right]\left[\begin{array}{c}
e_{6}^{T}-e_{4}^{T} \\
e_{3}^{T}-e_{6}^{T}
\end{array}\right], \\
\Xi_{2}= & -\left(e_{3}-e_{4}\right) E^{T} Z_{1} E\left(e_{3}-e_{4}\right)^{T} \\
& -\left[\begin{array}{c}
e_{6}^{T}-e_{5}^{T} \\
e_{4}^{T}-e_{6}^{T}
\end{array}\right]^{T}\left[\begin{array}{ll}
E^{T} Z_{2} E & E^{T} M_{2} E \\
E^{T} M_{2}^{T} E & E^{T} Z_{2} E
\end{array}\right]\left[\begin{array}{c}
e_{6}^{T}-e_{5}^{T} \\
e_{4}^{T}-e_{6}^{T}
\end{array}\right],
\end{aligned}
$$

and $\Psi \in \mathbf{R}^{n \times(n-r)}$ is any full-column rank matrix satisfying $E^{T} \Psi=0, e_{i}=\left[\begin{array}{lll}0_{n \times(i-1) n} & I_{n} & 0_{n \times(6-i) n}\end{array}\right]^{T}(i=1,2, \ldots, 6)$ are block entry matrices, and $e_{0}=\left(A e_{1}^{T}+A_{d} e_{6}^{T}\right)^{T}$.

Proof. The proof is divided into two parts. The first one deals with the regularity and impulse-free property, and the second one treats the stability property of the studied class of systems. Let us first of all show that the singular time-delay system (1) is regular and impulse-free. Using (5), it is easy to see that the following inequality holds:

$$
\begin{gathered}
E^{T} P A+A^{T} P E+U \Psi^{T} A+A^{T} \Psi U^{T}+\left(\frac{d_{1}}{2}\right)^{2} A^{T}\left(S_{1}+S_{2}\right) A \\
+\left(\frac{d_{2}-d_{1}}{2}\right)^{2} A^{T}\left(Z_{1}+Z_{2}\right) A+Q_{1}-E^{T} S_{1} E<0 .
\end{gathered}
$$

Using the fact that $S_{i}>0, Z_{i}>0(i=1,2)$, and $Q_{1}>0$, from (8), we have

$$
E^{T} P A+A^{T} P E+U \Psi^{T} A+A^{T} \Psi U^{T}-E^{T} S_{1} E<0 .
$$

It follows from (9) and Lemma 3.5 in [32] that the pair $(E, A)$ is regular and impulse-free. Thus, according to Definition 2, singular time-delay system (1) is regular and impulse-free.

Next, we will show the stability of the singular time-delay system (1). To the end, the following Lyapunov-Krasovskii functional for system (1) is considered:

$$
V\left(x_{t}\right)=\sum_{i=1}^{4} V_{i}\left(x_{t}\right)
$$

where

$$
\begin{aligned}
V_{1}\left(x_{t}\right)= & x^{T}(t) E^{T} P E x(t)+\int_{t-d(t)}^{t-d_{1}} x^{T}(s) W x(s) \mathrm{d} s, \\
V_{2}\left(x_{t}\right)= & \int_{t-\left(d_{1} / 2\right)}^{t} \xi_{1}^{T}(s) Q \xi_{1}(s) \mathrm{d} s \\
& +\int_{t-\left(\left(d_{1}+d_{2}\right) / 2\right)}^{t-d_{1}} \xi_{2}^{T}(s) R \xi_{2}(s) \mathrm{d} s, \\
V_{3}\left(x_{t}\right)= & \frac{d_{1}}{2}\left(\int_{-d_{1} / 2}^{0} \int_{t+\alpha}^{t} \dot{x}^{T}(\beta) E^{T} S_{1} E \dot{x}(\beta) \mathrm{d} \beta \mathrm{d} \alpha\right. \\
& \left.\quad+\int_{-d_{1}}^{-d_{1} / 2} \int_{t+\alpha}^{t} \dot{x}^{T}(\beta) E^{T} S_{2} E \dot{x}(\beta) \mathrm{d} \beta \mathrm{d} \alpha\right),
\end{aligned}
$$

$$
\begin{aligned}
V_{4}\left(x_{t}\right)= & \frac{d_{2}-d_{1}}{2} \\
& \times\left(\int_{-\left(d_{1}+d_{2}\right) / 2}^{-d_{1}} \int_{t+\alpha}^{t} \dot{x}^{T}(\beta) E^{T} Z_{1} E \dot{x}(\beta) \mathrm{d} \beta \mathrm{d} \alpha\right. \\
& \left.\quad+\int_{-d_{2}}^{-\left(d_{1}+d_{2}\right) / 2} \int_{t+\alpha}^{t} \dot{x}^{T}(\beta) E^{T} Z_{2} E \dot{x}(\beta) \mathrm{d} \beta \mathrm{d} \alpha\right),
\end{aligned}
$$

with

$$
\begin{aligned}
& \xi_{1}(s)=\left[\begin{array}{ll}
x^{T}(s) & x^{T}\left(s-\frac{d_{1}}{2}\right)
\end{array}\right]^{T}, \\
& \xi_{2}(s)=\left[\begin{array}{ll}
x^{T}(s) & x^{T}\left(s-\frac{d_{2}-d_{1}}{2}\right)
\end{array}\right]^{T} .
\end{aligned}
$$

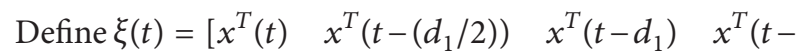
$\left.\left.\left(\left(d_{1}+d_{2}\right) / 2\right)\right) \quad x^{T}\left(t-d_{2}\right) \quad x^{T}(t-d(t))\right]^{T}$. Now calculating the derivative of $V\left(x_{t}\right)$ along the trajectory of the system (1), we derive

$$
\begin{aligned}
\dot{V}_{1}\left(x_{t}\right)= & 2 x^{T}(t) E^{T} P E \dot{x}(t)+x^{T}\left(t-d_{1}\right) W x\left(t-d_{1}\right) \\
& -(1-\dot{d}(t)) x^{T}(t-d(t)) W x(t-d(t)) \\
\leqslant & \xi^{T}(t)\left(e_{1} E^{T} P e_{0}^{T}+e_{0} P E e_{1}^{T}+e_{3} W e_{3}^{T}\right. \\
& \left.-(1-\mu) e_{6} W e_{6}^{T}\right) \xi(t), \\
\dot{V}_{2}\left(x_{t}\right)= & \xi_{1}^{T}(t) Q \xi_{1}(t)-\xi_{1}^{T}\left(t-\frac{d_{1}}{2}\right) Q \xi_{1}\left(t-\frac{d_{1}}{2}\right) \\
& +\xi_{2}^{T}\left(t-d_{1}\right) R \xi_{2}\left(t-d_{1}\right)-\xi_{2}^{T}\left(t-\frac{d_{1}+d_{2}}{2}\right) \\
& \times R \xi_{2}\left(t-\frac{d_{1}+d_{2}}{2}\right)
\end{aligned}
$$




$$
\begin{aligned}
= & \xi^{T}(t)\left(\left[\begin{array}{ll}
e_{1} & e_{2}
\end{array}\right] Q\left[\begin{array}{ll}
e_{1} & e_{2}
\end{array}\right]^{T}-\left[\begin{array}{ll}
e_{2} & e_{3}
\end{array}\right]\right. \\
& \times Q\left[\begin{array}{ll}
e_{2} & e_{3}
\end{array}\right]^{T}+\left[\begin{array}{ll}
e_{3} & e_{4}
\end{array}\right] R\left[\begin{array}{ll}
e_{3} & e_{4}
\end{array}\right]^{T} \\
& \left.-\left[\begin{array}{ll}
e_{4} & e_{5}
\end{array}\right] R\left[\begin{array}{ll}
e_{4} & e_{5}
\end{array}\right]^{T}\right) \xi(t) \\
\dot{V}_{3}\left(x_{t}\right)= & \left(\frac{d_{1}}{2}\right)^{2} \dot{x}^{T}(t) E^{T}\left(S_{1}+S_{2}\right) E \dot{x}(t) \\
& -\frac{d_{1}}{2} \int_{t-\left(d_{1} / 2\right)}^{t} \dot{x}^{T}(s) E^{T} S_{1} E \dot{x}(s) \mathrm{d} s \\
& -\frac{d_{1}}{2} \int_{t-d_{1}}^{t-\left(d_{1} / 2\right)} \dot{x}^{T}(s) E^{T} S_{2} E \dot{x}(s) \mathrm{d} s
\end{aligned}
$$

By Lemma 3, it can be shown that

$$
\begin{aligned}
\dot{V}_{3}\left(x_{t}\right) \leqslant & \left(\frac{d_{1}}{2}\right)^{2} \dot{x}^{T}(t) E^{T}\left(S_{1}+S_{2}\right) E \dot{x}(t) \\
& -\left(x(t)-x\left(t-\frac{d_{1}}{2}\right)\right)^{T} \\
& \times E^{T} S_{1} E\left(x(t)-x\left(t-\frac{d_{1}}{2}\right)\right) \\
& -\left(x\left(t-\frac{d_{1}}{2}\right)-x\left(t-d_{1}\right)\right)^{T} E^{T} S_{2} E \\
& \times\left(x\left(-\frac{d_{1}}{2}\right)-x\left(t-d_{1}\right)\right) \\
= & \xi^{T}(t)\left(\left(\frac{d_{1}}{2}\right)^{2} e_{0}\left(S_{1}+S_{2}\right) e_{0}^{T}\right. \\
& -\frac{d_{2}-d_{1}}{2} \int_{t-d_{2}}^{t-\left(\left(d_{1}+d_{2}\right) / 2\right)} \dot{x}^{T}(s) E^{T} Z_{2} E \dot{x}(s) \mathrm{d} s . \dot{x}^{T}(s) E^{T} Z_{1} E \dot{x}(s) \mathrm{d} s \\
\dot{V}_{4}\left(x_{t}\right)= & \left.\frac{d_{2}-d_{1}}{2}\right)^{2} \dot{x}^{T}(t) E^{T}\left(Z_{1}+Z_{2}\right) E \dot{x}(t) E^{T} S_{1} E\left(e_{1}-e_{2}\right)^{T} \\
& \left.-\left(e_{2}-e_{3}\right) E^{T} S_{2} E\left(e_{2}-e_{3}\right)^{T}\right) \xi(t),
\end{aligned}
$$

When $d_{1} \leqslant d(t) \leqslant\left(d_{1}+d_{2}\right) / 2$, we have

$$
\begin{aligned}
\dot{V}_{4}\left(x_{t}\right)= & \left(\frac{d_{2}-d_{1}}{2}\right)^{2} \dot{x}^{T}(t) E^{T}\left(Z_{1}+Z_{2}\right) E \dot{x}(t) \\
& -\frac{d_{2}-d_{1}}{2} \int_{t-d_{2}}^{t-\left(\left(d_{1}+d_{2}\right) / 2\right)} \dot{x}^{T}(s) E^{T} Z_{2} E \dot{x}(s) \mathrm{d} s
\end{aligned}
$$

$$
\begin{aligned}
& -\frac{d_{2}-d_{1}}{2} \int_{t-\left(\left(d_{1}+d_{2}\right) / 2\right)}^{t-d(t)} \dot{x}^{T}(s) E^{T} Z_{1} E \dot{x}(s) \mathrm{d} s \\
& -\frac{d_{2}-d_{1}}{2} \int_{t-d(t)}^{t-d_{1}} \dot{x}^{T}(s) E^{T} Z_{1} E \dot{x}(s) \mathrm{d} s .
\end{aligned}
$$

Using Lemma 3 again, we get

$$
\begin{aligned}
\dot{V}_{4}\left(x_{t}\right) \leqslant & \left(\frac{d_{2}-d_{1}}{2}\right)^{2} \dot{x}^{T}(t) E^{T}\left(Z_{1}+Z_{2}\right) E \dot{x}(t) \\
& -\left(x\left(t-\frac{d_{1}+d_{2}}{2}\right)-x\left(t-d_{2}\right)\right)^{T} E^{T} Z_{2} E \\
& \times\left(x\left(t-\frac{d_{1}+d_{2}}{2}\right)-x\left(t-d_{2}\right)\right) \\
& -\left(1+\frac{2\left(d(t)-d_{1}\right)}{d_{1}+d_{2}-2 d(t)}\right) \\
& \times\left(x(t-d(t))-x\left(t-\frac{d_{1}+d_{2}}{2}\right)\right)^{T} E^{T} Z_{1} E \\
& \times\left(x(t-d(t))-x\left(t-\frac{d_{1}+d_{2}}{2}\right)\right) \\
& -\left(1+\frac{d_{1}+d_{2}-2 d(t)}{2\left(d(t)-d_{1}\right)}\right) \\
& \times\left(x\left(t-d_{1}\right)-x(t-d(t))\right)^{T} E^{T} Z_{1} E \\
& \times\left(x\left(t-d_{1}\right)-x(t-d(t))\right) \\
& \\
& \\
& (x)
\end{aligned}
$$

Define

$$
\begin{aligned}
J=[ & \sqrt{\frac{2\left(d(t)-d_{1}\right)}{d_{1}+d_{2}-2 d(t)}} \\
& \times\left(x(t-d(t))-x\left(t-\frac{d_{1}+d_{2}}{2}\right)\right)^{T} E^{T} \\
& \left.-\sqrt{\frac{d_{1}+d_{2}-2 d(t)}{2\left(d(t)-d_{1}\right)}}\left(x\left(t-d_{1}\right)-x(t-d(t))\right)^{T} E^{T}\right],
\end{aligned}
$$

with the condition (6); then, premultiplying and postmultiplying $\left[\begin{array}{cc}Z_{1} & M_{1} \\ M_{1}^{T} & Z_{1}\end{array}\right]>0$ by $J$ and $J^{T}$, respectively, we can obtain

$$
\begin{gathered}
-\frac{2\left(d(t)-d_{1}\right)}{d_{1}+d_{2}-2 d(t)}\left(x(t-d(t))-x\left(t-\frac{d_{1}+d_{2}}{2}\right)\right)^{T} \\
\times E^{T} Z_{1} E\left(x(t-d(t))-x\left(t-\frac{d_{1}+d_{2}}{2}\right)\right) \\
-\frac{d_{1}+d_{2}-2 d(t)}{2\left(d(t)-d_{1}\right)}\left(x\left(t-d_{1}\right)-x(t-d(t))\right)^{T}
\end{gathered}
$$




$$
\begin{aligned}
& \times E^{T} Z_{1} E\left(x\left(t-d_{1}\right)-x(t-d(t))\right) \\
\leqslant & -\left(x(t-d(t))-x\left(t-\frac{d_{1}+d_{2}}{2}\right)\right)^{T} \\
& \times E^{T} M_{1} E\left(x\left(t-d_{1}\right)-x(t-d(t))\right) \\
& -\left(x\left(t-d_{1}\right)-x(t-d(t))\right)^{T} E^{T} M_{1}^{T} E \\
& \times\left(x(t-d(t))-x\left(t-\frac{d_{1}+d_{2}}{2}\right)\right)
\end{aligned}
$$

hence,

$$
\begin{aligned}
& \dot{V}_{4}\left(x_{t}\right) \leqslant\left(\frac{d_{2}-d_{1}}{2}\right)^{2} \dot{x}^{T}(t) E^{T}\left(Z_{1}+Z_{2}\right) E \dot{x}(t) \\
& -\left(x\left(t-\frac{d_{1}+d_{2}}{2}\right)-x\left(t-d_{2}\right)\right)^{T} E^{T} Z_{2} E \\
& \times\left(x\left(t-\frac{d_{1}+d_{2}}{2}\right)-x\left(t-d_{2}\right)\right) \\
& -\left[\begin{array}{c}
x(t-d(t))-x\left(t-\frac{d_{1}+d_{2}}{2}\right) \\
x\left(t-d_{1}\right)-x(t-d(t))
\end{array}\right]^{T} \\
& \times\left[\begin{array}{cc}
E^{T} Z_{1} E & E^{T} M_{1} E \\
E^{T} M_{1}^{T} E & E^{T} Z_{1} E
\end{array}\right] \\
& \times\left[\begin{array}{c}
x(t-d(t))-x\left(t-\frac{d_{1}+d_{2}}{2}\right) \\
x\left(t-d_{1}\right)-x(t-d(t))
\end{array}\right] \\
& =\xi^{T}(t)\left(\left(\frac{d_{2}-d_{1}}{2}\right)^{2} e_{0}\left(Z_{1}+Z_{2}\right) e_{0}^{T}\right. \\
& -\left(e_{4}-e_{5}\right) E^{T} Z_{2} E \\
& \times\left(e_{4}-e_{5}\right)^{T}-\left[\begin{array}{r}
e_{6}^{T}-e_{4}^{T} \\
e_{3}^{T}-e_{6}^{T}
\end{array}\right]^{T} \\
& \times\left[\begin{array}{cc}
E^{T} Z_{1} E & E^{T} M_{1} E \\
E^{T} M_{1}^{T} E & E^{T} Z_{1} E
\end{array}\right] \\
& \left.\times\left[\begin{array}{c}
e_{6}^{T}-e_{4}^{T} \\
e_{3}^{T}-e_{6}^{T}
\end{array}\right]\right) \xi(t) .
\end{aligned}
$$

Note that when $d(t)=d_{1}$ or $d(t)=\left(d_{1}+d_{2}\right) / 2$, we have $\xi^{T}(t)\left(e_{3}-e_{6}\right)=0$ or $\xi^{T}(t)\left(e_{6}-e_{4}\right)=0$, respectively. So relation (20) still holds.
When $\left(d_{1}+d_{2}\right) / 2 \leqslant d(t) \leqslant d_{2}$, (15) can be rewritten as

$$
\begin{aligned}
\dot{V}_{4}\left(x_{t}\right)= & \left(\frac{d_{2}-d_{1}}{2}\right)^{2} \dot{x}^{T}(t) E^{T}\left(Z_{1}+Z_{2}\right) E \dot{x}(t) \\
& -\frac{d_{2}-d_{1}}{2} \int_{t-\left(\left(d_{1}+d_{2}\right) / 2\right)}^{t-d_{1}} \dot{x}^{T}(s) E^{T} Z_{1} E \dot{x}(s) \mathrm{d} s \\
& -\frac{d_{2}-d_{1}}{2} \int_{t-d_{2}}^{t-d(t)} \dot{x}^{T}(s) E^{T} Z_{2} E \dot{x}(s) \mathrm{d} s \\
& -\frac{d_{2}-d_{1}}{2} \int_{t-d(t)}^{t-\left(\left(d_{1}+d_{2}\right) / 2\right)} \dot{x}^{T}(s) E^{T} Z_{2} E \dot{x}(s) \mathrm{d} s .
\end{aligned}
$$

From condition (6) and Lemma 3, proceeding in a similar manner as above, we can get

$$
\begin{aligned}
\dot{V}_{4}\left(x_{t}\right) \leqslant \xi^{T}(t)( & \left(\frac{d_{2}-d_{1}}{2}\right)^{2} e_{0}\left(Z_{1}+Z_{2}\right) e_{0}^{T} \\
& -\left(e_{3}-e_{4}\right) E^{T} Z_{1} E\left(e_{3}-e_{4}\right)^{T} \\
& -\left[\begin{array}{c}
e_{6}^{T}-e_{5}^{T} \\
e_{4}^{T}-e_{6}^{T}
\end{array}\right]\left[\begin{array}{ll}
E^{T} Z_{2} E & E^{T} M_{2} E \\
E^{T} M_{2}^{T} E & E^{T} Z_{2} E
\end{array}\right] \\
& \left.\times\left[\begin{array}{c}
e_{6}^{T}-e_{5}^{T} \\
e_{4}^{T}-e_{6}^{T}
\end{array}\right]\right) \xi(t) .
\end{aligned}
$$

Noting $E^{T} \Psi=0$, we can deduce

$$
0=2 x^{T}(t) U \Psi^{T} E \dot{x}(t)=2 \xi^{T}(t) e_{1} U \Psi^{T} e_{0}^{T} \xi(t),
$$

where $U$ is any matrix with appropriate dimensions.

Combining (10)-(14), (20)-(23) yield

$$
\dot{V}\left(x_{t}\right) \leqslant \begin{cases}\xi^{T}(t)\left(\Xi+\Xi_{1}\right) \xi(t), & d_{1} \leqslant d(t) \leqslant \frac{d_{1}+d_{2}}{2}, \\ \xi^{T}(t)\left(\Xi+\Xi_{2}\right) \xi(t), & \frac{d_{1}+d_{2}}{2} \leqslant d(t) \leqslant d_{2} .\end{cases}
$$

Therefore, we can see if LMIs (5) and (6) hold for any $k \epsilon$ $\{1,2\}$; then $\dot{V}\left(x_{t}\right)<0$ holds for any $d(t) \in\left[d_{1}, d_{2}\right]$. Hence, there exist a sufficiently small scalar $\varepsilon>0$, such that

$$
\dot{V}(t) \leqslant-\varepsilon\|x(t)\|^{2} .
$$

By (25), the following steps are similar to the proofs of Theorem 3.1 in [25] and Proposition 1 in [28]; we deduce that singular time-delay system (1) is stable. This completes the proof.

Remark 5. Based on the Lyapunov-Krasovskii functional (10), Theorem 4 proposed a new delay-dependent criterion guaranteeing the singular time-delay system (1) to be regular, impulse-free, and stable. Lyapunov-Krasovskii functional 
(10) is constructed by using the idea of "delay decomposition" [28-30]. We decompose the delay intervals $\left[0, d_{1}\right]$ and $\left[d_{1}, d_{2}\right]$ into two equidistant subintervals, respectively, such that the information of delay states $x\left(t-\left(d_{1} / 2\right)\right)$ and $x\left(t-\left(\left(d_{1}+d_{2}\right) / 2\right)\right)$ is all taken into account. Compared with [29], in which the Lyapunov-Krasovskii functional was constructed by decomposing delay intervals $\left[0, d_{1}\right]$ and $\left[0, d_{2}\right]$ into two equidistant subintervals, respectively, our Lyapunov-Krasovskii functional contains the delay state $x(t-$ $\left.\left(\left(d_{1}+d_{2}\right) / 2\right)\right)$. Thus, by checking the variation of $\dot{V}\left(x_{t}\right)$ for the case when $d(t) \in\left[d_{1},\left(d_{1}+d_{2}\right) / 2\right]$ or $d(t) \in\left[\left(d_{1}+\right.\right.$ $\left.\left.d_{2}\right) / 2, d_{2}\right]$, respectively, we introduce a new estimation on the upper bound of $\dot{V}\left(x_{t}\right)$. That is to say, the delay state $x\left(t-\left(\left(d_{1}+d_{2}\right) / 2\right)\right)$ plays a key role in the further reduction of conservatism. Furthermore, our Lyapunov-Krasovskii functional can be easily extended to the case as the number of delay partitions grows, which can be seen from the following discussion.

In the following, by decomposing the delay intervals $\left[0, d_{1}\right]$ and $\left[d_{1}, d_{2}\right]$ into $N(N \geqslant 2)$ equidistant subintervals, respectively, we will derive a more general stability result than Theorem 4. For this purpose, the following LyapunovKrasovskii functional for singular time-delay system (1) is considered:

$$
\begin{aligned}
& \widetilde{V}\left(x_{t}\right)= x^{T}(t) E^{T} P E x(t)+\int_{t-d(t)}^{t-d_{1}} x^{T}(s) W x(s) \mathrm{d} s \\
&+\sum_{i=1}^{N-1}\left(\int_{t-(i / N) d_{1}}^{t-((i-1) / N) d_{1}} \eta_{1}^{T}(s) Q_{i} \eta_{1}(s) \mathrm{d} s\right. \\
&\left.+\int_{t-d_{1}-(i / N)\left(d_{2}-d_{1}\right)}^{t-d_{1}-((i-1) / N)\left(d_{2}-d_{1}\right)} \eta_{2}^{T}(s) R_{i} \eta_{2}(s) \mathrm{d} s\right) \\
&+\sum_{i=1}^{N}\left(\frac{d_{1}}{N} \int_{-(i / N) d_{1}}^{-((i-1) / N) d_{1}} \int_{t+\alpha}^{t} \dot{x}^{T}(\beta) E^{T}\right. \\
&+\frac{d_{2}-d_{1}}{N} \int_{-d_{1}-(i / N)\left(d_{2}-d_{1}\right)}^{-d_{1}-((i-1) / N)\left(d_{2}-d_{1}\right)} \times S_{i} E \dot{x}(\beta) \mathrm{d} \beta \mathrm{d} \alpha \\
& \times \int_{t+\alpha}^{t} \dot{x}^{T}(\beta) E^{T} Z_{i} E \dot{x}
\end{aligned}
$$

where

$$
\begin{aligned}
& \eta_{1}(s)=\left[x^{T}(s) x^{T}\left(s-\frac{1}{N} d_{1}\right)\right]^{T}, \\
& \eta_{2}(s)=\left[x^{T}(s) x^{T}\left(s-\frac{1}{N}\left(d_{2}-d_{1}\right)\right)\right]^{T},
\end{aligned}
$$

$$
\begin{gathered}
P>0, \quad W>0, \quad Q_{i}=\left[\begin{array}{ll}
Q_{i 1} & Q_{i 2} \\
Q_{i 2}^{T} & Q_{i 3}
\end{array}\right]>0, \\
R_{i}=\left[\begin{array}{cc}
R_{i 1} & R_{i 2} \\
R_{i 2}^{T} & R_{i 3}
\end{array}\right]>0 \quad(i=1,2, \ldots, N-1), \\
S_{j}>0, \quad Z_{j}>0 \quad(j=1,2, \ldots, N) .
\end{gathered}
$$

Using Lyapunov-Krasovskii functional (26), the following theorem can be obtained.

Theorem 6. Given an integer $N \geqslant 2$ and scalars $0<d_{1}<d_{2}$, $0 \leqslant \mu<1$, for any delay $d(t)$ satisfying (2), singular timedelay system (1) is regular, impulse-free, and stable if there exist matrices

$$
\begin{gathered}
P>0, \quad W>0, \quad Q_{i}=\left[\begin{array}{ll}
Q_{i 1} & Q_{i 2} \\
Q_{i 2}^{T} & Q_{i 3}
\end{array}\right]>0, \\
R_{i}=\left[\begin{array}{ll}
R_{i 1} & R_{i 2} \\
R_{i 2}^{T} & R_{i 3}
\end{array}\right]>0 \quad(i=1,2, \ldots, N-1), \\
S_{j}>0, \quad Z_{j}>0, \quad M_{j}(j=1,2, \ldots, N)
\end{gathered}
$$

and $U$, such that LMIs

$$
\Gamma+\Gamma_{k}<0,
$$

$$
\left[\begin{array}{cc}
Z_{k} & M_{k} \\
M_{k}^{T} & Z_{k}
\end{array}\right]>0
$$

hold for any $k \in\{1,2, \ldots, N\}$, where

$$
\begin{aligned}
& \Gamma=e_{1}\left(E^{T} P+U \Psi^{T}\right) e_{0}^{T}+e_{0}\left(P E+\Psi U^{T}\right) e_{1}^{T} \\
& +e_{N+1} W e_{N+1}^{T}-(1-\mu) e_{2 N+2} W e_{2 N+2}^{T} \\
& +\sum_{i=1}^{N-1}\left(\left[\begin{array}{ll}
e_{i} & e_{i+1}
\end{array}\right] Q_{i}\left[\begin{array}{ll}
e_{i} & e_{i+1}
\end{array}\right]^{T}\right. \\
& -\left[\begin{array}{ll}
e_{i+1} & e_{i+2}
\end{array}\right] Q_{i}\left[\begin{array}{ll}
e_{i+1} & e_{i+2}
\end{array}\right]^{T} \\
& +\left[\begin{array}{ll}
e_{N+i} & e_{N+i+1}
\end{array}\right] R_{i}\left[\begin{array}{ll}
e_{N+i} & e_{N+i+1}
\end{array}\right]^{T} \\
& \left.-\left[\begin{array}{ll}
e_{N+i+1} & e_{N+i+2}
\end{array}\right] R_{i}\left[\begin{array}{ll}
e_{N+i+1} & e_{N+i+2}
\end{array}\right]^{T}\right) \\
& +\sum_{i=1}^{N}\left(e_{0}\left(\left(\frac{d_{1}}{N}\right)^{2} S_{i}+\left(\frac{d_{2}-d_{1}}{N}\right)^{2} Z_{i}\right) e_{0}^{T}\right. \\
& -\left(e_{i}-e_{i+1}\right) E^{T} S_{i} E\left(e_{i}-e_{i+1}\right)^{T} \\
& \left.-\left(e_{N+i}-e_{N+i+1}\right) E^{T} Z_{i} E\left(e_{N+i}-e_{N+i+1}\right)^{T}\right),
\end{aligned}
$$




$$
\begin{aligned}
\Gamma_{k}= & \left(e_{N+k}-e_{N+k+1}\right) E^{T} Z_{k} E\left(e_{N+k}-e_{N+k+1}\right)^{T} \\
& -\left[\begin{array}{c}
e_{2 N+2}^{T}-e_{N+k+1}^{T} \\
e_{N+k}^{T}-e_{2 N+2}^{T}
\end{array}\right]^{T}\left[\begin{array}{cc}
E^{T} Z_{k} E & E^{T} M_{k} E \\
E^{T} M_{k}^{T} E & E^{T} Z_{k} E
\end{array}\right] \\
& \times\left[\begin{array}{c}
e_{2 N+2}^{T}-e_{N+k+1}^{T} \\
e_{N+k}^{T}-e_{2 N+2}^{T}
\end{array}\right],
\end{aligned}
$$

and $\Psi \in \mathbf{R}^{n \times(n-r)}$ is any full-column rank matrix satisfying $E^{T} \Psi=0, e_{i}=\left[\begin{array}{lll}0_{n \times(i-1) n} & I_{n} & 0_{n \times(2 N-i+2) n}\end{array}\right]^{T}(i=1,2, \ldots, 2 N+$ 2) are block entry matrices, and $e_{0}=\left(A e_{1}^{T}+A_{d} e_{2 N+2}^{T}\right)^{T}$.

Proof. The proof of Theorem 6 can be carried out using methods in the proof of Theorem 4 . Hence, it is omitted.

Remark 7. Based on the Lyapunov-Krasovskii functional (26), Theorem 6 proposed an improved delay-dependent criterion guaranteeing the singular time-delay system (1) to be regular, impulse-free, and stable. Lyapunov-Krasovskii functional (26) is constructed by decomposing the delay intervals $\left[0, d_{1}\right]$ and $\left[d_{1}, d_{2}\right]$ into $N$ equidistant subintervals, respectively, such that the information of delay states $x(t-$ $\left.(i / N) d_{1}\right)$ and $x\left(t-d_{1}-(i / N)\left(d_{2}-d_{1}\right)\right)(i=1,2, \ldots, N)$ is all taken into account. Compared with $[28,30]$, the augmented vectors $\eta_{1}(s)$ and $\eta_{2}(s)$ were introduced in LyapunovKrasovskii functional (26), so (26) is more general than the ones in $[28,30]$.

Remark 8. If the integer $N$ is set to 2 , then Theorem 6 reduces to Theorem 4 . That is, Theorem 6 is an extension of Theorem 4 . It should be pointed out that the conservatism of Theorem 6 lies in the parameter $N$, which refers to the number of delay partitioning; that is, the conservatism is reduced as the partitions grow. On the other hand, the computational complexity also depends on the partition number $N$; that is, the computational complexity is increased as the partitions become thinner. Generally taking the small value of $N$ such as $N=2$ and $N=3$, we can obtain less conservative and simple results. This can be seen from the simulation results in the sequel.

For system (1) with the routine delay case described by

$$
0 \leqslant d \leqslant(t) d, \quad \dot{d}(t) \leqslant \mu,
$$

where $d>0$ and $0 \leqslant \mu<1$ are known constant scalars, the corresponding Lyapunov-Krasovskii reduces to

$$
\begin{aligned}
\bar{V}\left(x_{t}\right)= & x^{T}(t) E^{T} P E x(t)+\int_{t-d(t)}^{t} x^{T}(s) W x(s) \mathrm{d} s \\
& +\sum_{i=1}^{N-1} \int_{t-(i / N) d}^{t-((i-1) / N) d} \zeta^{T}(s) R_{i} \zeta(s) \mathrm{d} s \\
& +\frac{d}{N} \sum_{i=1}^{N} \int_{-(i / N) d}^{-((i-1) / N) d} \int_{t+\alpha}^{t} \dot{x}^{T}(\beta) E^{T} Z_{i} E \dot{x}(\beta) \mathrm{d} \beta \mathrm{d} \alpha,
\end{aligned}
$$

where

$$
\begin{gathered}
\zeta(s)=\left[x^{T}(s) x^{T}\left(s-\frac{1}{N} d\right)\right]^{T}, \quad P>0, \quad W>0, \\
R_{i}=\left[\begin{array}{cc}
R_{i 1} & R_{i 2} \\
R_{i 2}^{T} & R_{i 3}
\end{array}\right]>0 \quad(i=1,2, \ldots, N-1), \\
Z_{j}>0 \quad(j=1,2, \ldots, N) .
\end{gathered}
$$

Similar to the proof of Theorem 4, we can obtain the following delay-dependent stability criterion for singular time-delay system (1) with $d(t)$ satisfying (32).

Corollary 9. Given an integer $N \geqslant 2$ and scalars $d>0$, $0 \leqslant \mu<1$, for any delay $d(t)$ satisfying (32), singular timedelay system (1) is regular, impulse-free, and stable if there exist matrices

$$
\begin{gathered}
P>0, \quad W>0, \\
R_{i}=\left[\begin{array}{ll}
R_{i 1} & R_{i 2} \\
R_{i 2}^{T} & R_{i 3}
\end{array}\right]>0 \quad(i=1,2, \ldots, N-1), \\
Z_{j}>0, \quad M_{j}(j=1,2, \ldots, N),
\end{gathered}
$$

and $U$, such that the LMI (30) and LMI

$$
\Upsilon+\Upsilon_{k}<0
$$

hold for any $k \in\{1,2, \ldots, N\}$, where

$$
\begin{aligned}
& \Upsilon=e_{1}\left(E^{T} P+U \Psi^{T}\right) e_{0}^{T}+e_{0}\left(P E+\Psi U^{T}\right) e_{1}^{T} \\
& +e_{1} W e_{1}^{T}-(1-\mu) e_{N+2} W e_{N+2}^{T} \\
& +\sum_{i=1}^{N-1}\left(\left[\begin{array}{ll}
e_{i} & e_{i+1}
\end{array}\right] R_{i}\left[\begin{array}{ll}
e_{i} & e_{i+1}
\end{array}\right]^{T}\right. \\
& \left.-\left[\begin{array}{ll}
e_{i+1} & e_{i+2}
\end{array}\right] R_{i}\left[\begin{array}{ll}
e_{i+1} & e_{i+2}
\end{array}\right]^{T}\right) \\
& +\sum_{i=1}^{N}\left(\left(\frac{d}{N}\right)^{2} e_{0} Z_{i} e_{0}^{T}-\left(e_{i}-e_{i+1}\right) E^{T} Z_{i} E\left(e_{i}-e_{i+1}\right)^{T}\right), \\
& \Upsilon_{k}=\left(e_{k}-e_{k+1}\right) E^{T} Z_{k} E\left(e_{k}-e_{k+1}\right)^{T}-\left[\begin{array}{c}
e_{N+2}^{T}-e_{k+1}^{T} \\
e_{k}^{T}-e_{N+2}^{T}
\end{array}\right]^{T} \\
& \times\left[\begin{array}{cc}
E^{T} Z_{k} E & E^{T} M_{k} E \\
E^{T} M_{k}^{T} E & E^{T} Z_{k} E
\end{array}\right]\left[\begin{array}{c}
e_{N+2}^{T}-e_{k+1}^{T} \\
e_{k}^{T}-e_{N+2}^{T}
\end{array}\right],
\end{aligned}
$$

and $\Psi \in \mathbf{R}^{n \times(n-r)}$ is any full-column rank matrix satisfying $E^{T} \Psi=0, e_{i}=\left[\begin{array}{lll}0_{n \times(i-1) n} & I_{n} & 0_{n \times(N-i+2) n}\end{array}\right]^{T}(i=1,2, \ldots, N+2)$ are block entry matrices, and $e_{0}=\left(A e_{1}^{T}+A_{d} e_{N+2}^{T}\right)^{T}$.

Remark 10. Theorem 6 and Corollary 9 give stability criteria of system (1) with $d(t)$ satisfying (2) and (32), respectively. It is noted that the conditions in Theorem 6 and 
Corollary 9 are both delay-dependent and rate-dependent. However, the information of delay rate may not be known in many cases, or $d(t)$ even may not be differentiable; then Theorem 6 and Corollary 9 fail to work. Regarding these circumstances, delay-dependent and rate-independent criteria can be derived by choosing $W=0$ in Theorem 6 and Corollary 9.

For the case when $d(t)$ satisfies $0<d_{1} \leqslant d(t) \leqslant d_{2}$ and $\mu$ is unknown, the following result can be obtained from Theorem 6 by setting $W=0$.

Corollary 11. Given an integer $N \geqslant 2$ and scalars $0<d_{1}<d_{2}$, for any delay $d(t)$ satisfying $d_{1} \leqslant d(t) \leqslant d_{2}$, singular timedelay system (1) is regular, impulse-free, and stable if there exist matrices

$$
\begin{gathered}
P>0, \quad Q_{i}=\left[\begin{array}{ll}
Q_{i 1} & Q_{i 2} \\
Q_{i 2}^{T} & Q_{i 3}
\end{array}\right]>0, \\
R_{i}=\left[\begin{array}{ll}
R_{i 1} & R_{i 2} \\
R_{i 2}^{T} & R_{i 3}
\end{array}\right]>0 \quad(i=1,2, \ldots, N-1), \\
S_{j}>0, \quad Z_{j}>0, \quad M_{j}(j=1,2, \ldots, N),
\end{gathered}
$$

and $U$, such that the LMI (30) and LMI

$$
\Gamma_{0}+\Gamma_{k}<0
$$

hold for any $k \in\{1,2, \ldots, N\}$, where

$$
\begin{aligned}
\Gamma_{0}=e_{1}\left(E^{T} P\right. & \left.+U \Psi^{T}\right) e_{0}^{T}+e_{0}\left(P E+\Psi U^{T}\right) e_{1}^{T} \\
+ & \sum_{i=1}^{N-1}\left(\left[\begin{array}{ll}
e_{i} & e_{i+1}
\end{array}\right] Q_{i}\left[\begin{array}{ll}
e_{i} & e_{i+1}
\end{array}\right]^{T}\right. \\
& -\left[\begin{array}{ll}
e_{i+1} & e_{i+2}
\end{array}\right] Q_{i}\left[\begin{array}{ll}
e_{i+1} & e_{i+2}
\end{array}\right]^{T} \\
& +\left[\begin{array}{ll}
e_{N+i} & e_{N+i+1}
\end{array}\right] R_{i}\left[e_{N+i} e_{N+i+1}\right]^{T} \\
& \left.-\left[\begin{array}{ll}
e_{N+i+1} & e_{N+i+2}
\end{array}\right] R_{i}\left[e_{N+i+1} e_{N+i+2}\right]^{T}\right) \\
+\sum_{i=1}^{N}( & \left(\begin{array}{ll}
\left.\left.\frac{d_{1}}{N}\right)^{2} S_{i}+\left(\frac{d_{2}-d_{1}}{N}\right)^{2} Z_{i}\right) e_{0}^{T} \\
N
\end{array}\right. \\
& -\left(e_{i}-e_{i+1}\right) E^{T} S_{i} E\left(e_{i}-e_{i+1}\right)^{T} \\
& \left.-\left(e_{N+i}-e_{N+i+1}\right) E^{T} Z_{i} E\left(e_{N+i}-e_{N+i+1}\right)^{T}\right)
\end{aligned}
$$

and $\Gamma_{k}, \Psi, e_{i}(i=0,1,2, \ldots, 2 N+2)$ are the same as those defined in Theorem 6.

For the case when $d(t)$ satisfies $0 \leqslant d(t) \leqslant d$ and $\mu$ is unknown, the following result can be obtained from Corollary 9 by setting $W=0$.
Corollary 12. Given an integer $N \geqslant 2$ and scalar $d>0$, for any delay $d(t)$ satisfying $0 \leqslant d(t) \leqslant d$, singular timedelay system (1) is regular, impulse-free, and stable if there exist matrices

$$
\begin{gathered}
P>0, \quad R_{i}=\left[\begin{array}{cc}
R_{i 1} & R_{i 2} \\
R_{i 2}^{T} & R_{i 3}
\end{array}\right]>0 \quad(i=1,2, \ldots, N-1), \\
Z_{j}>0, \quad M_{j}(j=1,2, \ldots, N),
\end{gathered}
$$

and $U$, such that the LMI (30) and LMI

$$
\Upsilon_{0}+\Upsilon_{k}<0
$$

hold for any $k \in\{1,2, \ldots, N\}$, where

$$
\begin{aligned}
& \Upsilon_{0}=e_{1}\left(E^{T} P+U \Psi^{T}\right) e_{0}^{T}+e_{0}\left(P E+\Psi U^{T}\right) e_{1}^{T} \\
& +\sum_{i=1}^{N-1}\left(\left[\begin{array}{ll}
e_{i} & e_{i+1}
\end{array}\right] R_{i}\left[\begin{array}{ll}
e_{i} & e_{i+1}
\end{array}\right]^{T}-\left[\begin{array}{ll}
e_{i+1} & e_{i+2}
\end{array}\right]\right. \\
& \left.\quad \times R_{i}\left[\begin{array}{ll}
e_{i+1} & e_{i+2}
\end{array}\right]^{T}\right) \\
& +\sum_{i=1}^{N}\left(\left(\frac{d}{N}\right)^{2} e_{0} Z_{i} e_{0}^{T}-\left(e_{i}-e_{i+1}\right) E^{T} Z_{i} E\left(e_{i}-e_{i+1}\right)^{T}\right),
\end{aligned}
$$

and $\Upsilon_{k}, \Psi, e_{i}(i=0,1,2, \ldots, N+2)$ are the same as those defined in Corollary 9.

Remark 13. Compared with the results in [20-25], the LMIs in Theorems 4 and 6 and Corollaries 9 and 11, 12 are all strict LMIs; thus, they can be directly solved by using any LMI toolbox like the one of Matlab or the one of Scilab.

When the matrix $E$ is nonsingular, the stability problem of singular time-delay system (1) is reduced to analyzing the stability of the following regular system:

$$
\begin{aligned}
& \dot{x}(t)=A x(t)+A_{d} x(t-d(t)), \\
& x(t)=\varphi(t), \quad t \in\left[-d_{2}, 0\right] .
\end{aligned}
$$

This problem has been widely studied in the recent literature (see, e.g., [7-15]). Choose the following Lyapunov-Krasovskii functional:

$$
\begin{aligned}
\widetilde{\widetilde{V}}\left(x_{t}\right)= & x^{T}(t) P x(t)+\int_{t-d(t)}^{t-d_{1}} x^{T}(s) W x(s) \mathrm{d} s \\
+ & \sum_{i=1}^{N-1}\left(\int_{t-(i / N) d_{1}}^{t-((i-1) / N) d_{1}} \eta_{1}^{T}(s) Q_{i} \eta_{1}(s) \mathrm{d} s\right. \\
& \left.+\int_{t-d_{1}-(i / N)\left(d_{2}-d_{1}\right)}^{t-d_{1}-((i-1) / N)\left(d_{2}-d_{1}\right)} \eta_{2}^{T}(s) R_{i} \eta_{2}(s) \mathrm{d} s\right)
\end{aligned}
$$


TABLE 1: Allowable upper bound $d_{2}$ with $d_{1}=0$ for different $\mu$.

\begin{tabular}{lccc}
\hline Methods & $\mu=0$ & $\mu=0.3$ & $\mu=0.7$ \\
\hline Zhong and Yang [20] & 0.5567 & - & - \\
Wu and Zhou [22] & 1.0660 & - & - \\
Li and Jia [24] & 1.0660 & 1.0130 & 0.9510 \\
Lin and Fei [28] & 1.0660 & 1.0260 & 1.0220 \\
Corollary 9 $(N=2)$ & 1.0937 & 1.0597 & 1.0597 \\
Corollary 9 $(N=3)$ & 1.1000 & 1.0689 & 1.0689 \\
\hline
\end{tabular}

TABLE 2: Allowable upper bound $d_{2}$ with given $d_{1}$ for different $\mu$.

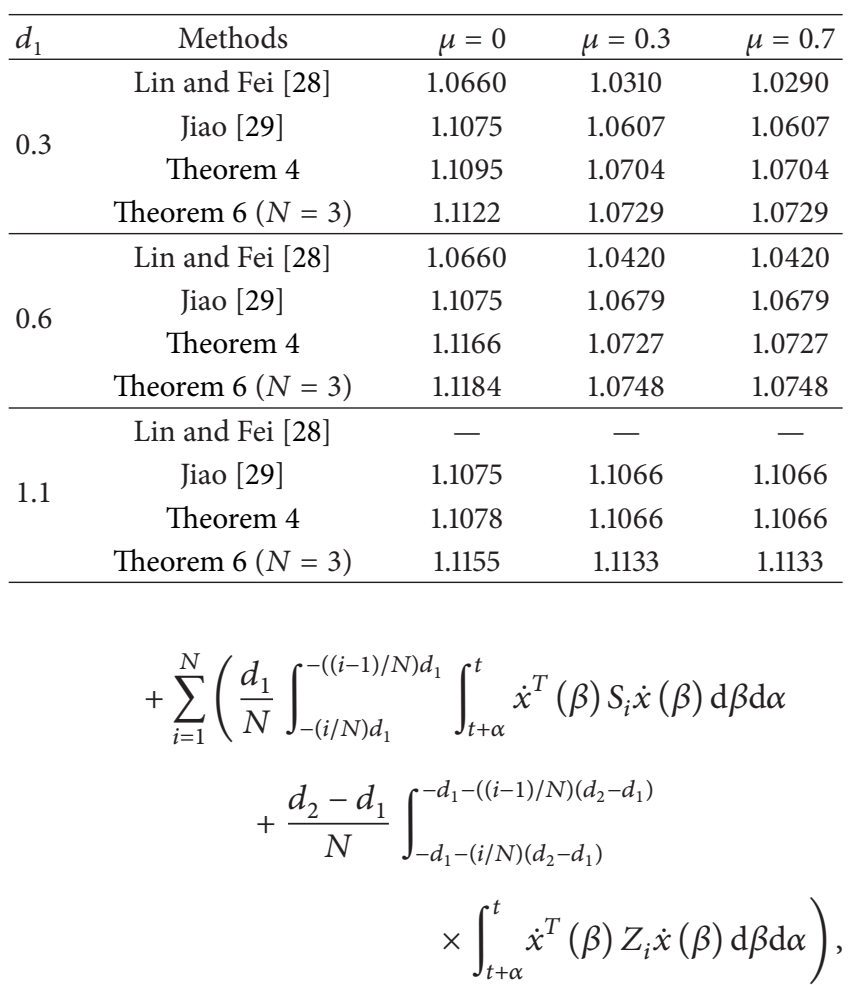

where vectors $\eta_{1}(s), \eta_{2}(s)$ and matrices $P, W, Q_{i}, R_{i}(i=$ $1,2, \ldots, N-1), S_{j}, Z_{j}(j=1,2, \ldots, N)$ are defined the same as those defined in (26).

By employing the Lyapunov-Krasovskii functional (45) and using the similar proof of Theorem 4, we can obtain the following delay-dependent stability criterion for time-delay system (44).

Corollary 14. Given an integer $N \geqslant 2$ and scalars $0<d_{1}<$ $d_{2}, 0 \leqslant \mu<1$, for any delay $d(t)$ satisfying (2), time-delay system (44) is stable if there exist matrices

$$
\begin{gathered}
P>0, \quad W>0, \quad Q_{i}=\left[\begin{array}{ll}
Q_{i 1} & Q_{i 2} \\
Q_{i 2}^{T} & Q_{i 3}
\end{array}\right]>0, \\
R_{i}=\left[\begin{array}{ll}
R_{i 1} & R_{i 2} \\
R_{i 2}^{T} & R_{i 3}
\end{array}\right]>0 \quad(i=1,2, \ldots, N-1), \\
S_{j}>0, \quad Z_{j}>0, \quad M_{j}(j=1,2, \ldots, N),
\end{gathered}
$$

such that the LMI (30) and LMI (47)

$$
\widetilde{\Gamma}+\widetilde{\Gamma}_{k}<0
$$

hold for any $k \in\{1,2, \ldots, N\}$, where

$$
\begin{aligned}
& \widetilde{\Gamma}=e_{1} P e_{0}^{T}+e_{0} P e_{1}^{T}+e_{N+1} W e_{N+1}^{T}-(1-\mu) e_{2 N+2} W e_{2 N+2}^{T} \\
& +\sum_{i=1}^{N-1}\left(\left[\begin{array}{ll}
e_{i} & e_{i+1}
\end{array}\right] Q_{i}\left[\begin{array}{ll}
e_{i} & e_{i+1}
\end{array}\right]^{T}-\left[\begin{array}{ll}
e_{i+1} & e_{i+2}
\end{array}\right]\right. \\
& \times Q_{i}\left[\begin{array}{ll}
e_{i+1} & e_{i+2}
\end{array}\right]^{T}+\left[\begin{array}{ll}
e_{N+i} & e_{N+i+1}
\end{array}\right] R_{i} \\
& \times\left[\begin{array}{ll}
e_{N+i} & e_{N+i+1}
\end{array}\right]^{T}-\left[\begin{array}{ll}
e_{N+i+1} & e_{N+i+2}
\end{array}\right] R_{i} \\
& \left.\times\left[\begin{array}{ll}
e_{N+i+1} & e_{N+i+2}
\end{array}\right]^{T}\right) \\
& +\sum_{i=1}^{N}\left(e_{0}\left(\left(\frac{d_{1}}{N}\right)^{2} S_{i}+\left(\frac{d_{2}-d_{1}}{N}\right)^{2} Z_{i}\right) e_{0}^{T}\right. \\
& -\left(e_{i}-e_{i+1}\right) S_{i}\left(e_{i}-e_{i+1}\right)^{T} \\
& \left.-\left(e_{N+i}-e_{N+i+1}\right) Z_{i}\left(e_{N+i}-e_{N+i+1}\right)^{T}\right) \\
& \widetilde{\Gamma}_{k}=\left(e_{N+k}-e_{N+k+1}\right) Z_{k}\left(e_{N+k}-e_{N+k+1}\right)^{T} \\
& -\left[\begin{array}{c}
e_{2 N+2}^{T}-e_{N+k+1}^{T} \\
e_{N+k}^{T}-e_{2 N+2}^{T}
\end{array}\right]^{T} \\
& \times\left[\begin{array}{cc}
Z_{k} & M_{k} \\
M_{k}^{T} & Z_{k}
\end{array}\right]\left[\begin{array}{c}
e_{2 N+2}^{T}-e_{N+k+1}^{T} \\
e_{N+k}^{T}-e_{2 N+2}^{T}
\end{array}\right],
\end{aligned}
$$

and $e_{i}(i=0,1,2, \ldots, 2 N+2)$ are the same as those defined in Theorem 6.

Based on Corollary 9, we can get the following stability criterion for system (44) with delay $d(t)$ satisfying (32).

Corollary 15. Given an integer $N \geqslant 2$ and scalars $d>0,0 \leqslant$ $\mu<1$, for any delay $d(t)$ satisfying (32), time-delay system (44) is stable if there exist matrices

$$
P>0, \quad W>0
$$

$$
\begin{gathered}
R_{i}=\left[\begin{array}{ll}
R_{i 1} & R_{i 2} \\
R_{i 2}^{T} & R_{i 3}
\end{array}\right]>0 \quad(i=1,2, \ldots, N-1), \\
Z_{j}>0, \quad M_{j}(j=1,2, \ldots, N),
\end{gathered}
$$

such that the LMI (30) and LMI

$$
\widetilde{\Upsilon}+\widetilde{\Upsilon}_{k}<0
$$


hold for any $k \in\{1,2, \ldots, N\}$, where

$$
\begin{aligned}
\tilde{Y}= & e_{1} P e_{0}^{T}+e_{0} P e_{1}^{T}+e_{1} W e_{1}^{T}-(1-\mu) e_{N+2} W e_{N+2}^{T} \\
& +\sum_{i=1}^{N-1}\left(\begin{array}{ll}
e_{i} & e_{i+1}
\end{array}\right] R_{i}\left[\begin{array}{ll}
e_{i} & e_{i+1}
\end{array}\right]^{T} \\
& \left.-\left[\begin{array}{ll}
e_{i+1} & e_{i+2}
\end{array}\right] R_{i}\left[\begin{array}{ll}
e_{i+1} & e_{i+2}
\end{array}\right]^{T}\right) \\
& +\sum_{i=1}^{N}\left(\left(\frac{d}{N}\right)^{2} e_{0} Z_{i} e_{0}^{T}-\left(e_{i}-e_{i+1}\right) Z_{i}\left(e_{i}-e_{i+1}\right)^{T}\right) \\
\tilde{\Upsilon}_{k}= & \left(e_{k}-e_{k+1}\right) Z_{k}\left(e_{k}-e_{k+1}\right)^{T}-\left[e_{N+2}^{T}-e_{k+1}^{T}\right]^{T} \\
& \times\left[\begin{array}{ll}
Z_{k} & M_{k} \\
M_{k}^{T} & Z_{k}
\end{array}\right]\left[\begin{array}{l}
e_{N+2}^{T}-e_{k+1}^{T} \\
e_{k}^{T}-e_{N+2}^{T}
\end{array}\right],
\end{aligned}
$$

and $e_{i}(i=0,1,2, \ldots, N+2)$ are the same as those defined in Corollary 9.

When the information of $\mu$ is unknown, the following results can be obtained directly from Corollaries 11 and 12 for system (44) for two cases: $0<d_{1} \leqslant d(t) \leqslant d_{2}$ and $0 \leqslant d(t) \leqslant d$.

Corollary 16. Given an integer $N \geqslant 2$ and scalars $0<d_{1}<$ $d_{2}$, for any delay $d(t)$ satisfying $d_{1} \leqslant d(t) \leqslant d_{2}$, time-delay system (44) is stable if there exist matrices

$$
\begin{gathered}
P>0, \quad Q_{i}=\left[\begin{array}{ll}
Q_{i 1} & Q_{i 2} \\
Q_{i 2}^{T} & Q_{i 3}
\end{array}\right]>0, \\
R_{i}=\left[\begin{array}{ll}
R_{i 1} & R_{i 2} \\
R_{i 2}^{T} & R_{i 3}
\end{array}\right]>0 \quad(i=1,2, \ldots, N-1), \\
S_{j}>0, \quad Z_{j}>0, \quad M_{j}(j=1,2, \ldots, N),
\end{gathered}
$$

such that the LMI (30) and LMI

$$
\widetilde{\Gamma}_{0}+\widetilde{\Gamma}_{k}<0
$$

hold for any $k \in\{1,2, \ldots, N\}$, where

$$
\begin{aligned}
& \widetilde{\Gamma}_{0}=e_{1} P e_{0}^{T}+e_{0} P e_{1}^{T} \\
& +\sum_{i=1}^{N-1}\left(\left[\begin{array}{ll}
e_{i} & e_{i+1}
\end{array}\right] Q_{i}\left[\begin{array}{ll}
e_{i} & e_{i+1}
\end{array}\right]^{T}\right. \\
& -\left[\begin{array}{ll}
e_{i+1} & e_{i+2}
\end{array}\right] Q_{i}\left[\begin{array}{ll}
e_{i+1} & e_{i+2}
\end{array}\right]^{T} \\
& +\left[\begin{array}{ll}
e_{N+i} & e_{N+i+1}
\end{array}\right] R_{i}\left[e_{N+i} e_{N+i+1}\right]^{T} \\
& \left.-\left[\begin{array}{ll}
e_{N+i+1} & e_{N+i+2}
\end{array}\right] R_{i}\left[\begin{array}{ll}
e_{N+i+1} & e_{N+i+2}
\end{array}\right]^{T}\right) \\
& +\sum_{i=1}^{N}\left(e_{0}\left(\left(\frac{d_{1}}{N}\right)^{2} S_{i}+\left(\frac{d_{2}-d_{1}}{N}\right)^{2} Z_{i}\right) e_{0}^{T}\right. \\
& -\left(e_{i}-e_{i+1}\right) S_{i}\left(e_{i}-e_{i+1}\right)^{T} \\
& \left.-\left(e_{N+i}-e_{N+i+1}\right) Z_{i}\left(e_{N+i}-e_{N+i+1}\right)^{T}\right)
\end{aligned}
$$

$$
\begin{aligned}
\widetilde{\Gamma}_{k}= & \left(e_{N+k}-e_{N+k+1}\right) Z_{k}\left(e_{N+k}-e_{N+k+1}\right)^{T} \\
& -\left[\begin{array}{c}
e_{2 N+2}^{T}-e_{N+k+1}^{T} \\
e_{N+k}^{T}-e_{2 N+2}^{T}
\end{array}\right]^{T}\left[\begin{array}{ll}
Z_{k} & M_{k} \\
M_{k}^{T} & Z_{k}
\end{array}\right]\left[\begin{array}{c}
e_{2 N+2}^{T}-e_{N+k+1}^{T} \\
e_{N+k}^{T}-e_{2 N+2}^{T}
\end{array}\right],
\end{aligned}
$$

and $e_{i}(i=0,1,2, \ldots, 2 N+2)$ are the same as those defined in Theorem 6.

Corollary 17. Given an integer $N \geqslant 2$ and scalar $d>0$, for any delay $d(t)$ satisfying $0 \leqslant d(t) \leqslant d$, time-delay system (44) is stable if there exist matrices

$$
\begin{gathered}
P>0, \quad R_{i}=\left[\begin{array}{cc}
R_{i 1} & R_{i 2} \\
R_{i 2}^{T} & R_{i 3}
\end{array}\right]>0 \quad(i=1,2, \ldots, N-1), \\
Z_{j}>0, \quad M_{j}(j=1,2, \ldots, N),
\end{gathered}
$$

such that the LMI (30) and LMI

$$
\tilde{\Upsilon}_{0}+\tilde{\Upsilon}_{k}<0
$$

hold for any $k \in\{1,2, \ldots, N\}$, where

$$
\begin{aligned}
\tilde{\Upsilon}_{0}= & e_{1} P e_{0}^{T}+e_{0} P e_{1}^{T} \\
+ & +\sum_{i=1}^{N-1}\left(\left[\begin{array}{ll}
e_{i} & e_{i+1}
\end{array}\right] R_{i}\left[\begin{array}{ll}
e_{i} & e_{i+1}
\end{array}\right]^{T}\right. \\
& \left.-\left[\begin{array}{ll}
e_{i+1} & e_{i+2}
\end{array}\right] R_{i}\left[\begin{array}{ll}
e_{i+1} & e_{i+2}
\end{array}\right]^{T}\right)
\end{aligned}
$$


TABLE 3: Allowable upper bound $d_{2}$ with given $d_{1}$ for different $\mu$.

\begin{tabular}{ccccc}
\hline$d_{1}$ & Methods & $\mu=0.3$ & $\mu=0.5$ & $\mu=0.9$ \\
\hline & Zhang et al. [8] & 2.81 & 2.61 & 2.61 \\
$2 \quad$ Sun et al. [12] & 3.01 & 2.56 & 2.56 \\
& Qian and Liu [15] & 3.02 & 2.69 & 2.69 \\
& Corollary 14 $(N=2)$ & 3.18 & 2.75 & 2.75 \\
& Corollary 14 $(N=3)$ & 3.21 & 2.77 & 2.77 \\
\hline \multirow{4}{*}{$3 \quad$ Zhang et al. [8] } & 3.31 & 3.31 & 3.31 \\
& Sun et al. [12] & 3.34 & 3.34 & 3.34 \\
& Qian and Liu [15] & 3.41 & 3.41 & 3.41 \\
& Corollary 14 $(N=2)$ & 3.46 & 3.46 & 3.46 \\
& Corollary 14 $(N=3)$ & 3.48 & 3.48 & 3.48 \\
\hline \multirow{4}{*}{ Zhang et al. [8] } & 4.09 & 4.09 & 4.09 \\
& Sun et al. [12] & 4.16 & 4.16 & 4.16 \\
& Qian and Liu [15] & 4.20 & 4.20 & 4.20 \\
& Corollary 14 $(N=2)$ & 4.25 & 4.25 & 4.25 \\
& Corollary 14 $(N=3)$ & 4.28 & 4.28 & 4.28 \\
\hline \multirow{4}{*}{ Zhang et al. [8] } & - & - & - \\
& Sun et al. [12] & 5.02 & 5.02 & 5.02 \\
$5 \quad$ Qian and Liu [15] & 5.03 & 5.03 & 5.03 \\
& Corollary 14 $(N=2)$ & 5.09 & 5.09 & 5.09 \\
& Corollary 14 $(N=3)$ & 5.12 & 5.12 & 5.12 \\
\hline
\end{tabular}

$$
\begin{aligned}
& +\sum_{i=1}^{N}\left(\left(\frac{d}{N}\right)^{2} e_{0} Z_{i} e_{0}^{T}-\left(e_{i}-e_{i+1}\right) Z_{i}\left(e_{i}-e_{i+1}\right)^{T}\right), \\
\widetilde{\Upsilon}_{k}= & \left(e_{k}-e_{k+1}\right) Z_{k}\left(e_{k}-e_{k+1}\right)^{T} \\
& -\left[\begin{array}{c}
e_{N+2}^{T}-e_{k+1}^{T} \\
e_{k}^{T}-e_{N+2}^{T}
\end{array}\right]^{T}\left[\begin{array}{ll}
Z_{k} & M_{k} \\
M_{k}^{T} & Z_{k}
\end{array}\right]\left[\begin{array}{c}
e_{N+2}^{T}-e_{k+1}^{T} \\
e_{k}^{T}-e_{N+2}^{T}
\end{array}\right],
\end{aligned}
$$

and $e_{i}(i=0,1,2, \ldots, N+2)$ are the same as those defined in Corollary 9.

\section{Numerical Examples}

In the section, numerical examples are given to illustrate the effectiveness and the less conservatism of obtained results in this paper.

Example 18. Consider the singular time-delay system (1) with

$$
E=\left[\begin{array}{ll}
1 & 0 \\
0 & 0
\end{array}\right], \quad A=\left[\begin{array}{cc}
0.5 & 0 \\
0 & -1
\end{array}\right], \quad A_{d}=\left[\begin{array}{cc}
-1.1 & 1 \\
0 & 0.5
\end{array}\right]
$$

For $d_{1}=0$, the allowable upper bound $d_{2}$, which guarantees regular, impulse-free, and stable systems (1) for different $\mu$, is listed in Table 1. From Table 1, it can be seen that the stability criteria in Corollary 9 are less conservative than those in [20, $22,24,28]$.

For systems with interval time-varying delay, the allowable upper bound $d_{2}$, which guarantees regular, impulse-free,
TABLE 4: Number of decision variables.

\begin{tabular}{lc}
\hline Methods & Number of decision variables \\
\hline Zhang et al. [8] & $13 n^{2}+5 n$ \\
Sun et al. [12] & $18 n^{2}+8 n$ \\
Qian and Liu [15] & $22 n^{2}+8 n$ \\
Corollary 14 $(N=2)$ & $9 n^{2}+5 n$ \\
Corollary 14 $(N=3)$ & $15 n^{2}+8 n$ \\
\hline
\end{tabular}

and stable systems (1) with given lower bound $d_{1}$ for different $\mu$, is listed in Table 2. From Table 2, it can be seen that the stability criteria in Theorems 4 and 6 are less conservative than those in $[28,29]$. Especially, when $d_{1}=1.1$, the result in [28] is not feasible while the allowable upper bound $d_{2}$ can also be obtained from Theorems 4 and 6 in this paper.

Example 19. Consider the time-delay system (44) with

$$
A=\left[\begin{array}{cc}
-2 & 0 \\
0 & -0.9
\end{array}\right], \quad A_{d}=\left[\begin{array}{cc}
-1 & 0 \\
-1 & -1
\end{array}\right]
$$

This example is used in many recent papers such as [7-15, 29]. The purpose is to compare the allowable upper bound $d_{2}$ which guarantees the stability of the considered system for given lower bound $d_{1}$.

For various $\mu$, the allowable upper bound $d_{2}$ is listed in Table 3. Moreover, the number of decision variables involved in the stability criteria is given in Table 4. From Tables 3 and 4 , it can be seen that Corollary $14(N=2)$ in this paper has fewer decision variables and less conservatism than those results in $[8,12,15]$.

For unknown $\mu$, the allowable upper bound $d_{2}$ is listed in Table 5. From Table 5, it can be seen that Corollary 16 in this paper is less conservative than those results in $[7-15,29]$.

\section{Conclusion}

This paper deals with the problem of stability for singular systems with interval time-varying delay. By using a delay decomposition approach, new stability criteria for singular time-delay systems to be regular, impulse-free, and stable are proposed in terms of LMIs. Based on the obtained criterion, some improved stability results for the regular systems with interval time-varying delay are also given. The obtained results in this paper have been shown to be less conservative than recently reported results. Moreover, the proposed method decreases the computational complexity comparable to some existing methods. Two numerical examples are given to illustrate the applicability of the results.

\section{Conflict of Interests}

The authors declare that there is no conflict of interests regarding the publication of this paper. 
TABLE 5: Allowable upper bound $d_{2}$ with given $d_{1}$ for unknown $\mu$.

\begin{tabular}{|c|c|c|c|c|}
\hline Methods & $d_{1}=1$ & $d_{1}=2$ & $d_{1}=3$ & $d_{1}=4$ \\
\hline He et al. [7] & 1.74 & 2.43 & 3.22 & 4.06 \\
\hline Shao [9] & 1.76 & 2.50 & 3.25 & 4.07 \\
\hline Zhang et al. [8] and Park et al. [10] & 2.06 & 2.61 & 3.31 & 4.09 \\
\hline Sun et al. [12] & 1.90 & 2.56 & 3.34 & 4.16 \\
\hline Qian et al. [14] & 1.90 & 2.56 & 3.34 & 4.17 \\
\hline Sun et al. [11] & 1.90 & 2.56 & 3.41 & 4.17 \\
\hline Qian and Liu [15] & 2.09 & 2.69 & 3.41 & 4.20 \\
\hline Liu et al. [13] & 2.09 & 2.69 & 3.41 & 4.20 \\
\hline Jiao [29] & 2.12 & 2.72 & 3.45 & 4.25 \\
\hline Corollary $16(N=2)$ & 2.21 & 2.75 & 3.46 & 4.26 \\
\hline Corollary $16(N=3)$ & 2.25 & 2.77 & 3.48 & 4.28 \\
\hline
\end{tabular}

\section{Acknowledgments}

This work was supported by the scientific research program funded by Shaanxi Provincial Education Department (no. 2013JK0601) and the scientific research program funded by Shaanxi Baoji city (no. 2013R4-3).

\section{References}

[1] K. Gu, V. L. Kharitonov, and J. Chen, Stability of Time-Delay Systems, Birkhauser, Boston, Mass, USA, 2003.

[2] E. Fridman and U. Shaked, "An improved stabilization method for linear time-delay systems," IEEE Transactions on Automatic Control, vol. 47, no. 11, pp. 1931-1937, 2002.

[3] W. Qian, S. Cong, Y. Sun, and S. Fei, "Novel robust stability criteria for uncertain systems with time-varying delay," Applied Mathematics and Computation, vol. 215, no. 2, pp. 866-872, 2009.

[4] X. M. Zhang and Q. L. Han, "A delay decomposition approach to delay-dependent stability for linear systems with time-varying delays," International Journal of Robust and Nonlinear Control, vol. 19, no. 17, pp. 1922-1930, 2009.

[5] J. Kim, "Note on stability of linear systems with time-varying delay," Automatica, vol. 47, no. 9, pp. 2118-2121, 2011.

[6] P. Liu, "State feedback stabilization of time-varying delay uncertain systems: a delay decomposition approach," Linear Algebra and Its Applications, vol. 438, no. 5, pp. 2188-2209, 2013.

[7] Y. He, Q. Wang, C. Lin, and M. Wu, "Delay-range-dependent stability for systems with time-varying delay," Automatica, vol. 43, no. 2, pp. 371-376, 2007.

[8] W. Zhang, X. Cai, and Z. Han, "Robust stability criteria for systems with interval time-varying delay and nonlinear perturbations," Journal of Computational and Applied Mathematics, vol. 234, no. 1, pp. 174-180, 2010.

[9] H. Y. Shao, "New delay-dependent stability criteria for systems with interval delay," Automatica, vol. 45, no. 3, pp. 744-749, 2009.

[10] P. Park, J. W. Ko, and C. Jeong, "Reciprocally convex approach to stability of systems with time-varying delays," Automatica, vol. 47, no. 1, pp. 235-238, 2011.

[11] J. Sun, G. P. Liu, J. Chen, and D. Rees, "Improved stability criteria for linear systems with time-varying delay," IET Control Theory \& Applications, vol. 4, no. 4, pp. 683-689, 2010.
[12] J. Sun, G. P. Liu, J. Chen, and D. Rees, "Improved delay-rangedependent stability criteria for linear systems with time-varying delays," Automatica, vol. 46, no. 2, pp. 466-470, 2010.

[13] Y. Liu, L. Hu, and P. Shi, "A novel approach on stabilization for linear systems with time-varying input delay," Applied Mathematics and Computation, vol. 218, no. 10, pp. 5937-5947, 2012.

[14] W. Qian, S. Cong, T. Li, and S. Fei, "Improved stability conditions for systems with interval time-varying delay," International Journal of Control, Automation and Systems, vol. 10, no. 6, pp. 1146-1152, 2012.

[15] W. Qian and J. Liu, "New stability analysis for systems with interval time-varying delay," Journal of the Franklin Institute. Engineering and Applied Mathematics, vol. 350, no. 4, pp. 890897, 2013

[16] Y. Wang, A. Yu, and X. Zhang, "Robust stability of stochastic genetic regulatory networks with time-varying delays: a delay fractioning approach," Neural Computing and Applications, vol. 23, no. 5, pp. 1217-1227, 2013.

[17] Y. T. Wang, X. Zhang, and Y. He, "Improved delay-dependent robust stability criteria for a class of uncertain mixed neutral and Lur'e dynamical systems with interval time-varying delays and sector-bounded nonlinearity," Nonlinear Analysis: Real World Applications, vol. 13, no. 5, pp. 2188-2194, 2012.

[18] F. Li, L. Wu, and P. Shi, "Stochastic stability of semi-Markovian jump systems with mode-dependent delays," International Journal of Robust and Nonlinear Control, 2013.

[19] L. Dai, Singular Control Systems, Springer, Berlin, Germany, 1989.

[20] R. Zhong and Z. Yang, "Delay-dependent robust control of descriptor systems with time delay," Asian Journal of Control, vol. 8, no. 1, pp. 36-44, 2006.

[21] S. Zhou and W. X. Zheng, "Robust $\mathrm{H}_{\infty}$ control of delayed singular systems with linear fractional parametric uncertainties," Journal of the Franklin Institute, vol. 346, no. 2, pp. 147-158, 2009.

[22] Z. G. Wu and W. N. Zhou, "Delay-dependent robust $H_{\infty}$ control for uncertain singular time-delay systems," IET Control Theory \& Applications, vol. 1, no. 5, pp. 1234-1241, 2007.

[23] L. L. Liu, J. G. Peng, and B. W. Wu, " $H_{\infty}$ control of singular timedelay systems via discretized Lyapunov functional," Journal of the Franklin Institute, vol. 348, no. 4, pp. 749-762, 2011.

[24] L. Li and Y. M. Jia, "Observer-based resilient L2-L1 control for singular time-delay systems," IET Control Theory and Applications, vol. 3, no. 10, pp. 1351-1362, 2009. 
[25] E. Boukas, "Delay-dependent robust stabilizability of singular linear systems with delays," Stochastic Analysis and Applications, vol. 27, no. 4, pp. 637-655, 2009.

[26] F. Li and X. Zhang, "A delay-dependent bounded real lemma for singular LPV systems with time-variant delay," International Journal of Robust and Nonlinear Control, vol. 22, no. 5, pp. 559$574,2012$.

[27] J. Jiao, "A stability criterion for singular systems with two additive time-varying delay components," International Journal of Automation and Computing, vol. 10, no. 1, pp. 39-45, 2013.

[28] J. Lin and S. Fei, "Reliable control for a class of uncertain singular systems with interval time-varying delay," Asian Journal of Control, vol. 13, no. 4, pp. 542-552, 2011.

[29] J. Jiao, "Delay-dependent stability criteria for singular systems with interval time-varying delay," Mathematical Problems in Engineering, vol. 2012, Article ID 570834, 16 pages, 2012.

[30] F. Li and X. Zhang, "Delay-range-dependent robust $H_{\infty}$ filtering for singular LPV systems with time variant delay," International Journal of Innovative Computing, Information and Control, vol. 9, no. 1, pp. 339-353, 2013.

[31] J. Jiao, "Robust stability and stabilization of discrete singular systems with interval time-varying delay and linear fractional uncertainty," International Journal of Automation and Computing, vol. 9, no. 1, pp. 8-15, 2012.

[32] X. Zhang and H. Y. Zhu, "Robust stability and stabilization criteria for discrete singular time-delay LPV systems," Asian Journal of Control, vol. 14, no. 4, pp. 1084-1094, 2012.

[33] H. Zhu, X. Zhang, and S. Cui, "Further results on $H_{\infty}$ control for discrete-time uncertain singular systems with interval timevarying delays in state and input," Optimal Control Applications \& Methods, vol. 34, no. 3, pp. 328-347, 2013.

[34] F. B. Li, P. Shi, L. G. Wu et al., "Fuzzy-model-based D-Stability and non-fragile control for discrete-time descriptor systems with multiple delays," IEEE Transactions on Fuzzy Systems, 2013, 10.1109/TFUZZ.2013.227264 7. 


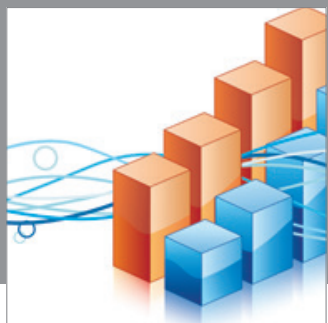

Advances in

Operations Research

mansans

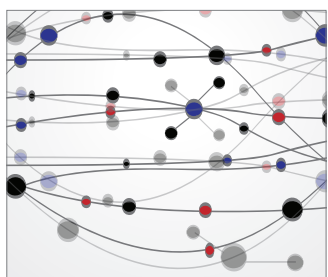

The Scientific World Journal
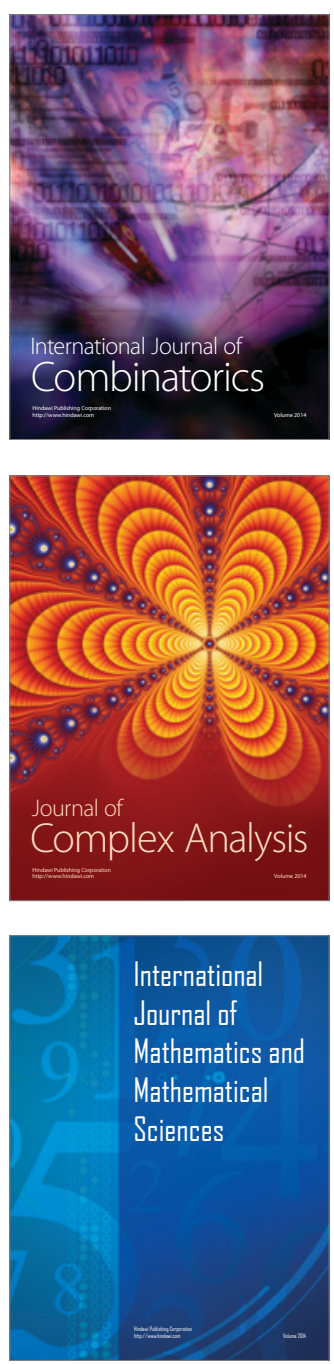
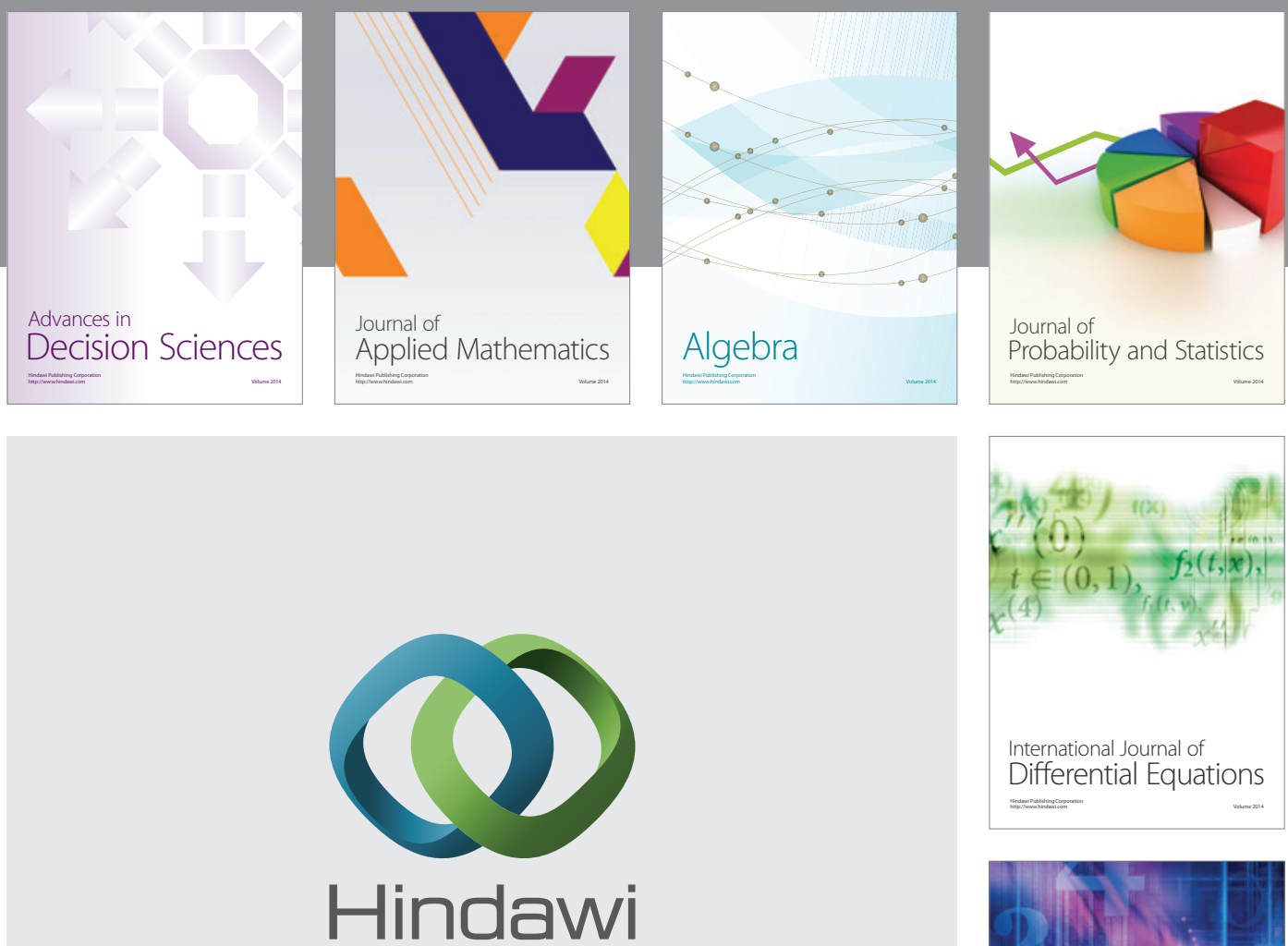

Submit your manuscripts at http://www.hindawi.com
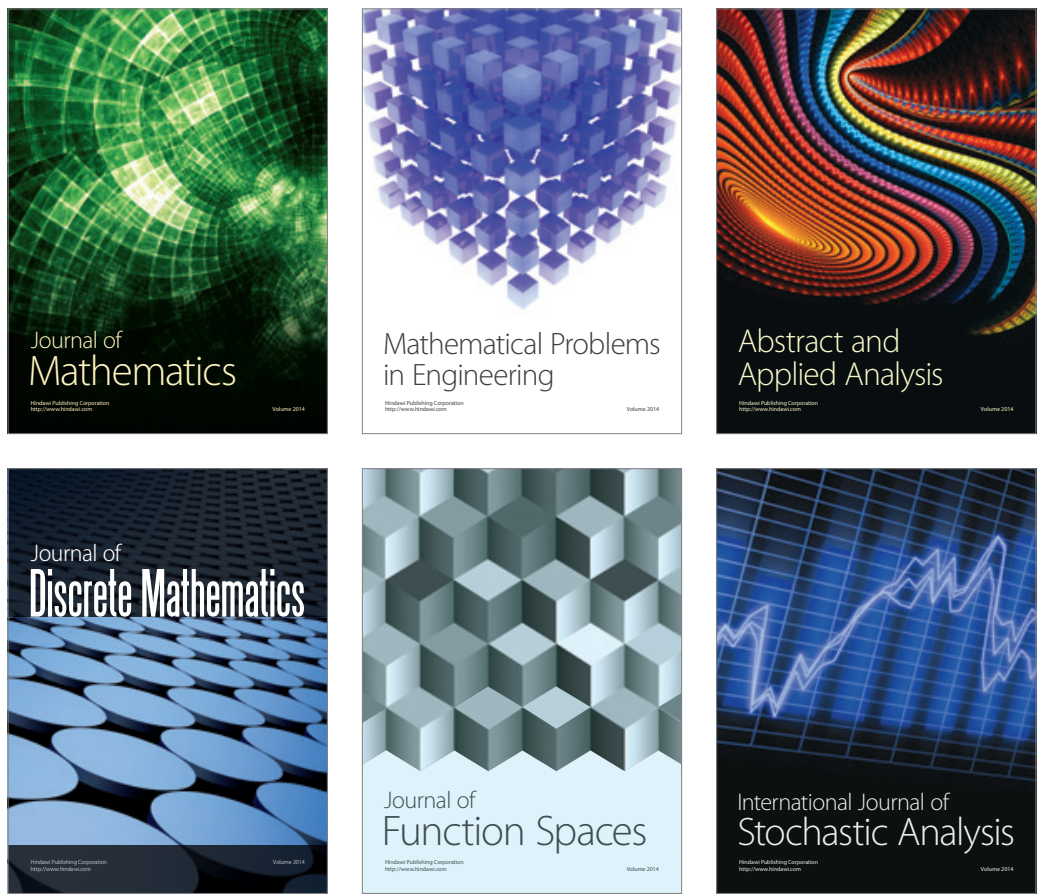

Journal of

Function Spaces

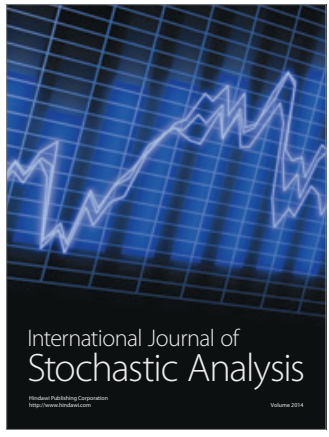

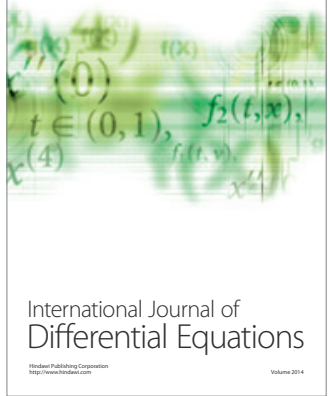
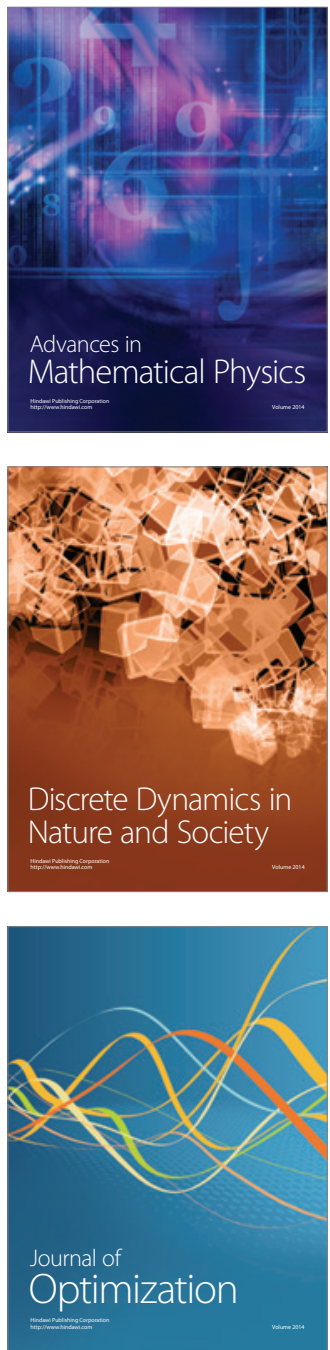